\title{
The Role of Central and Enteric Nervous Systems in the Control of the Retrograde Giant Contraction
}

\author{
Ivan M Lang \\ Dysphagia Animal Research Laboratory, Division of Gastroenterology and Hepatology, Medical College of Wisconsin, Milwaukee, WI, USA
}

\section{Background/Aims}

The role of the enteric (ENS) and central (CNS) nervous systems in the control of the retrograde giant contraction (RGC) associated with vomiting is unknown.

\section{Methods}

The effects of myotomy or mesenteric nerve transection (MNT) on apomorphine-induced emesis were investigated in 18 chronically instrumented dogs

\section{Results}

Neither surgery affected the RGC orad of the surgical site or the velocity of the RGC over the entire small intestine. Myotomy blocked the RGC for $17 \pm 5 \mathrm{~cm}$ aborad of the myotomy, and the velocity of the RGC from 100 to $70 \mathrm{~cm}$ from the pylorus slowed $(18.1 \pm$ 3.0 to $9.0 \pm 0.8 \mathrm{~cm} / \mathrm{sec}$ ) such that the RGC orad and aborad of the myotomy occurred simultaneously. After MNT, the RGC was unchanged up to $66 \pm 6 \mathrm{~cm}$ from the pylorus, and the sequence of the RGC across the denervated intestine was unaltered. The velocity of the RGC from 100 to $70 \mathrm{~cm}$ from the pylorus increased from $12.8 \pm 1.6$ to $196 \pm 116 \mathrm{~cm} / \mathrm{sec}$. After myotomy or MNT, the percent occurrence and magnitude of the RGC across the intestine 100 to $70 \mathrm{~cm}$ from the pylorus decreased.

\section{Conclusions}

The CNS activates the RGC 10 to $20 \mathrm{~cm}$ aborad of its innervation of the intestine and controls the RGC sequence. On the other hand, the ENS plays a role in initiation and generation of the RGC.

(J Neurogastroenterol Motil 2016;22:321-332)

\section{Key Words}

Central nervous system; Enteric nervous system; Vomiting

\section{Introduction}

Vomiting is associated with a series of gastrointestinal motor events ${ }^{1}$ which act to propel intestinal contents orad for subsequent expulsion and the primary motor event which propels intestinal contents into the stomach ${ }^{2,3}$ is the retrograde giant contraction (RGC). The RGC begins just prior to vomiting in the mid small intestine ${ }^{4,5}$ and it travels orad to the gastric corpus. ${ }^{1,5}$ Cervical vagotomy blocks activation of the RGC throughout the digestive tract ${ }^{5}$, and transec-

Received: August 31, 2015 Revised: October 24, 2015 Accepted: November 22, 2015

(a) This is an Open Access article distributed under the terms of the Creative Commons Attribution Non-Commercial License (http://creativecommons. org/licenses/by-nc/4.0) which permits unrestricted non-commercial use, distribution, and reproduction in any medium, provided the original work is properly cited.

*Correspondence: Ivan M Lang, DVM, PhD

Dysphagia Animal Research Laboratory, Division of Gastroenterology and Hepatology, Medical College of Wisconsin, 8701 Watertown Plank Road, Milwaukee, WI 53226, USA

Tel: +1-414-456-8138, Fax: +1-414-456-6215, E-mail: imlang@mcw.edu 
tion of the celiac branch of the vagus nerve blocks activation of the $\mathrm{RGC}^{6}$ in the small intestine only, therefore, the RGC is initiated by the central nervous system (CNS) through the vagus nerves, and propagation through the small intestine is dependent upon a neural pathway through the celiac ganglia. Vagal innervation of the small intestine is mediated by the celiac and mesenteric ganglia through the mesenteric nerves, ${ }^{7,8}$ therefore, CNS activation of the RGC through the small intestine is mediated by the mesenteric nerves.

A basic concept of the control of digestive tract motor activity is that all motor events are programmed by the enteric nervous system (ENS), but the RGC is clearly controlled to a certain extent by the CNS. The primacy of the CNS in the control of the RGC was investigated by sectioning the jejuno-ileum into 3 equal length segments and re-anastomosing them such that the segments were out of normal order. This surgery preserved activation of the normal propagation sequence of the $\mathrm{RGC},{ }^{10}$ suggesting that the $\mathrm{RGC}$ is controlled in a point by point manner by the CNS through the mesenteric nerves with little or no input from the ENS. On the other hand, Gregory ${ }^{11}$ indicates that the ENS does have a role in this process as the RGC is not observed in short Thiry-Vella loops of proximal jejunum during vomiting. The primary differences between these studies were the length of intestine surgically manipulated and the length over which the RGC response was recorded. In one case $^{10}$ intestinal segments of about $100 \mathrm{~cm}$ long with 2 recording sites were used, whereas in the other ${ }^{11}$ intestinal segments of about $15 \mathrm{~cm}$ long with one recording site were used. These studies suggest that while the CNS controls the RGC over the entire small intestine, the ENS participates in the local control over the RGC.

While the above studies suggest the relative roles of the ENS and CNS in the control of the RGC, no studies have experimentally investigated this issue. Therefore, the aim of this study was to determine the roles of the CNS and ENS in the control of the initiation, generation, and propagation of the RGC.

\section{Materials and Methods}

These experiments were performed at the Zablocki Veterans Affairs Medical Center and approved by the Animal Care and Use Committee of the Zablocki Veterans Affairs Medical Center. Eighteen mixed-breed dogs of either sex, 10 males and 8 females, weighing between $18 \mathrm{~kg}$ and $25 \mathrm{~kg}$ were implanted with recording devices on the muscles of the gastrointestinal tract using aseptic techniques as described previously. ${ }^{5,12,13}$ Recovery time of at least 20 days was allowed between surgeries, and experiments were conducted between 1 and 6 weeks after the neural interruption surger- ies described below.

\section{Animal Preparation}

\section{Gastrointestinal recording devices}

The dogs were anesthetized using pentobarbital sodium (30 $\mathrm{mg} / \mathrm{kg}, \mathrm{IV}$ ) and prepared for a sterile surgical procedure. A midventral laparotomy was made to expose the abdominal cavity, and sets of bipolar electrodes ( 6 dogs), force transducers ( 8 dogs), or both force transducer and electrodes (4 dogs) were sewn onto the seromuscular layer of the stomach and small intestine of each dog. Various arrangements of devices were used, and the following list includes the number of animals $(\mathrm{N})$ with devices in the listed location in cm from the pylorus: gastric antrum: 2 to $3 \mathrm{~cm}$ (18); duodenum: $15 \mathrm{~cm}$ (18); jejunum: $40 \mathrm{~cm}$ (18), $50 \mathrm{~cm}$ (18), 60 to $65 \mathrm{~cm}$ (7), 70 to $75 \mathrm{~cm}$ (14), 80 to $85 \mathrm{~cm}$ (15), $90 \mathrm{~cm}$ (13), 100 to $130 \mathrm{~cm}$ (18), 150 to $180 \mathrm{~cm}$ (12), 200 to $230 \mathrm{~cm}$ (10), 250 to $280 \mathrm{~cm} \mathrm{(9),}$ and ileum: 300 to $350 \mathrm{~cm}$ (8). The locations of the devices are listed in the text and figures according to the intestinal location, ie, duodenum (D), jejunum (J), or ileum (I), and distance from the pylorus, eg, D15 (in the duodenum $15 \mathrm{~cm}$ from the pylorus) and J150 (in the jejunum $150 \mathrm{~cm}$ from the pylorus). The poles of the electrodes were $7 \mathrm{~mm}$ apart and oriented in the longitudinal axis of the gastrointestinal tract. The force transducers were oriented to record contractions from the circular muscle. The devices were distributed along the digestive tract in order to monitor the motor activity of the entire small intestine. The devices were placed $10 \mathrm{~cm}$ apart in the surgical zone in order to record alterations in the RGC's as a result of the surgeries. The leads from the electrodes and force transducers were brought out of the abdomen through a stainless steel cannula as described previously., ${ }^{5,12,13}$

\section{Intravenous catheter}

A Silastic catheter was implanted in the jugular vein and the opposite end of each catheter was fitted with an IV catheter plug and implanted subcutaneously in the back of the neck. This catheter allowed for non-traumatic repeated administration of pharmacological agents intravenously. This catheter was kept patent by daily injection of heparinized saline $(20 \mathrm{U} / \mathrm{mL})$.

\section{Interruption of neural control of the retrograde giant contraction}

After recording control responses to an emetic agent, 2 types of surgical procedures were performed on the dogs designed to interrupt either CNS or ENS control of the RGC. 
Mesenteric Nerve Transection. As described previously the mesenteric nerves mediate the central control of the RGC, ${ }^{5-8}$ therefore, in order to interrupt the CNS control of the RGC, the mesenteric nerves were sectioned from $50 \pm 3 \mathrm{~cm}$ to $83 \pm 6 \mathrm{~cm}$ from the pylorus in 9 dogs. A mesenteric arcade of vessels was selected and separated to expose a segment that supplied about 30 $\mathrm{cm}$ of the proximal jejunum. The vascular branches between this segment, and the vascular arcades proximal and distal to this segment were tied and transected. All nonvascular attachments of the separated vascular array were visually identified, stripped from the vessels, and severed.

Myotomy. In order to interrupt the ENS control of the RGC, seromuscular myotomy (myenteric plexus transection) at $63 \pm 2$ $\mathrm{cm}$ from the pylorus was performed in 9 dogs. The seromuscular layer was incised circumferentially down to the level of the submucosa and a $1 \mathrm{~cm}$ wide segment of the seromuscular layer was excised. The edges of the seromuscular layer were not sewn together.

\section{Activation of the Retrograde Giant Contraction}

The RGC was activated by the intravenous administration of the emetic agent apomorphine ${ }^{5,12}$ at a dose of 2.5 to $10 \mu \mathrm{g} / \mathrm{kg}$ of dog body weight. The time delay between apomorphine administration and RGC response was close to 60 seconds but this timing is not illustrated in the figures as it has no bearing on the results. Apomorphine activates the RGC by stimulation of the chemoreceptive trigger zone ${ }^{1,14}$ by way of the brainstem emetic generator ${ }^{15}$ and the vagus nerves. ${ }^{5}$ Prior studies have found that the RGC is an all or none phenomenon which is not related to the dose of apomorphine. ${ }^{16-19}$

\section{Experimental Protocol}

After an overnight fast, spontaneous gastrointestinal contractile and myoelectrical activity were recorded for sufficient time to allow passage of one complete migrating motor complex (MMC). The RGC activating agent was then administered intravenously during Phase I or II of the MMC. Apomorphine was not administered more than once per day or more than every other day. RGC's were activated at least 3 times in each animal and the quantified variables were averages of these trials for each animal.

\section{Data Acquisition and Storage}

\section{Recording devices}

Digestive tract motor activity was recorded using strain gauges sewn onto the muscles. This method was chosen because strain gauges minimize artifact, can record specifically from the circular muscles of the intestine, and can be implanted for long term chronic use.

The strain gauge force transducers (EA-06-031DE-120 option SE; Micro-Measurements, Raleigh, NC, USA) were glued to thin heat tempered copper-beryllium shims shaped to the contour of the dog small intestine. The electrodes were composed of a pair of 15 gauge silver wires 2-3 mm long and $7 \mathrm{~mm}$ apart. Teflon coated silver plated copper wires were soldered to the strain gauges and electrodes. The solder points of the gauges and electrodes were electrically insulated with acrylic, and waterproofed using polysulfide. The gauges and electrodes were then embedded in Silastic for biocompatibility and to allow sewing to the gastrointestinal tract. The wires from the strain gauges and electrodes were placed in a 31 pin Amphenol plug and the plug was embedded and fixed in a stainless steel trans-abdominal cannula using dental acrylic. The cannula was waterproofed using a mixture of bees and paraffin wax. This cannula allowed the electrodes to be easily and non-traumatically connected to the preamplifiers of the data acquisition equipment.

\section{Data recording and storage}

Each strain gauge was connected electrically to a quarter Wheatstone bridge circuit before amplification by a DC amplifier with the high frequency filter set at $15 \mathrm{~Hz}$ (Grass Model 7P122, Warwick, RI, USA). The signals from the strain gauges and electrodes were fed into the preamplifiers. The strain gauge signals were high frequency filtered at $15 \mathrm{~Hz}$ and the electrical signals were bandpass filtered between $3 \mathrm{~Hz}$ and $35 \mathrm{~Hz}$. The amplified signals were stored on tape (Vetter Model D, Rebersburg, PA, USA) and later transferred to a computer using CODAS (Dataq Instruments, Akron, $\mathrm{OH}, \mathrm{USA}$ ) hardware and software. Only 8 channels could be recorded at a time, therefore, for each experiment the most appropriate devices were recorded. Occasionally, the baseline of the electrical recording shifted at greater amplitude than the phasic response and this baseline shift was removed using the derivative function of the Advanced CODAS software at a smoothing factor of 8 .

The RGC is correlated with 2 types of electrical responses ${ }^{12}$ : the RGC potential and electrical control activity (ECA) disruption (Fig. 1). The ECA disruption occurs as an elimination of ECA activity. The RGC potential is one or a few large amplitude electrical potentials that occurs during ECA disruption. The RGC potential is the electrical correlate of the very beginning of the RGC, however, there is no direct electrical correlate of the remainder of the RGC. Therefore, while it is possible to quantify the velocity of the RGC from electrical recording, it is not possible to quantify the 


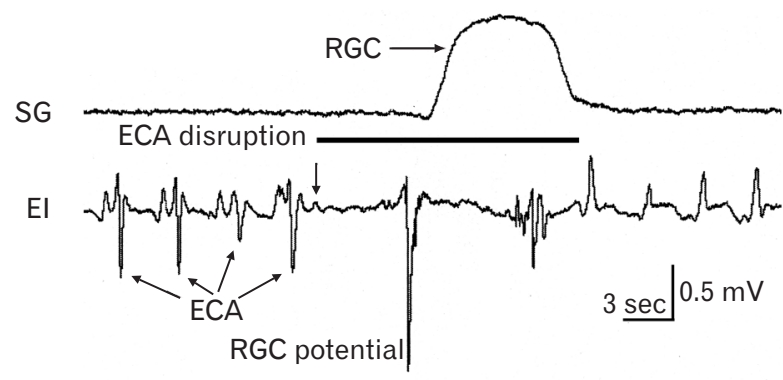

Figure 1. Myoelectrical correlates of retrograde giant contraction (RGC). This figure depicts the intestinal myoelectrical response (El) recorded from the same site as the strain gauge (SG) at $50 \mathrm{~cm}$ from the pylorus. Note that the electrical control activity (ECA) becomes disrupted, ie, ceases to occur (indicated by the arrow), about 5 seconds before the start of the RGC, and that the RGC potential occurs at the upstroke of the RGC.

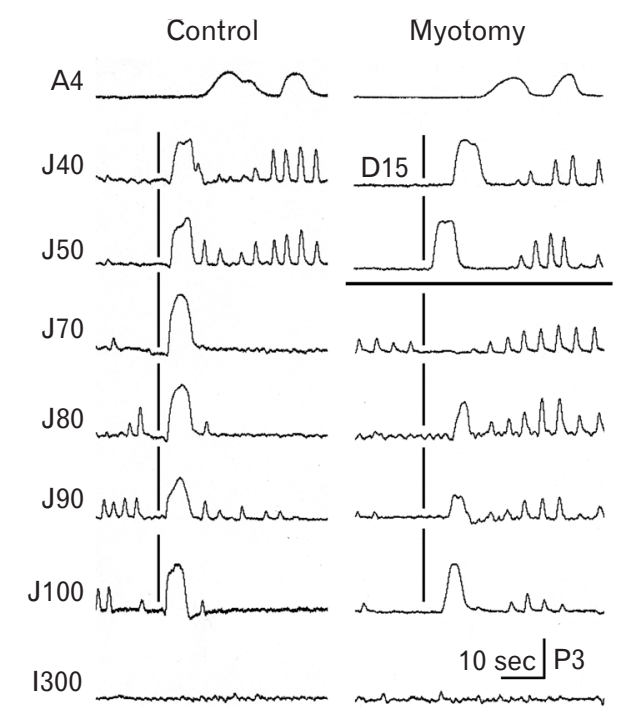

Figure 2. Effects of enteric nervous system (ENS) interruption on retrograde giant contraction (RGC). This figure depicts the effects of myotomy at $60 \mathrm{~cm}$ from the pylorus on the initiation and propagation of the RGC across the small intestine. Myotomy blocked occurrence of the RGC at J70, reduced the magnitude and duration of the RGC at J80, J90, and J100, and resulted in 2 separate RGC's occurring above and below the myotomy propagating independent of each other. Myotomy had no observable effects orad to the myotomy. The vertical lines in this figure are vertical reference lines so that direction and degree of propagation can be more easily assessed. The horizontal line in this figure depict the location of the myotomy. P3, phase III of the migrating motor complex; A, antrum; D, duodenum; J, jejunum; I, ileum. The numbers after the letters indicate the $\mathrm{cm}$ from pylorus of the strain gauge. magnitude or duration of the RGC based on electrical recordings. However, the magnitude and duration of the RGC was measured. The peak magnitude was quantified as percentage of the peak magnitude of phase III $^{19}$ contractions of the MMC. Strain gauge force measurements in chronic animals can change over time as the tissue accommodates to the devices. While this process may take many months to occur, it makes comparison of contractile forces over time unreliable. However, reliable force measurements can be made over time ${ }^{19}$ by quantifying the force relative to a very stable, reliable, and spontaneous contraction, ie, the phase III contractions of the MMC. Therefore, we quantified the RGC force as a percentage of the phase III contractile force.

\section{Statistical Methods}

The following variables were also quantified: RGC and RGC potential occurrence velocities, time delay to the jejunum at $50 \mathrm{~cm}$

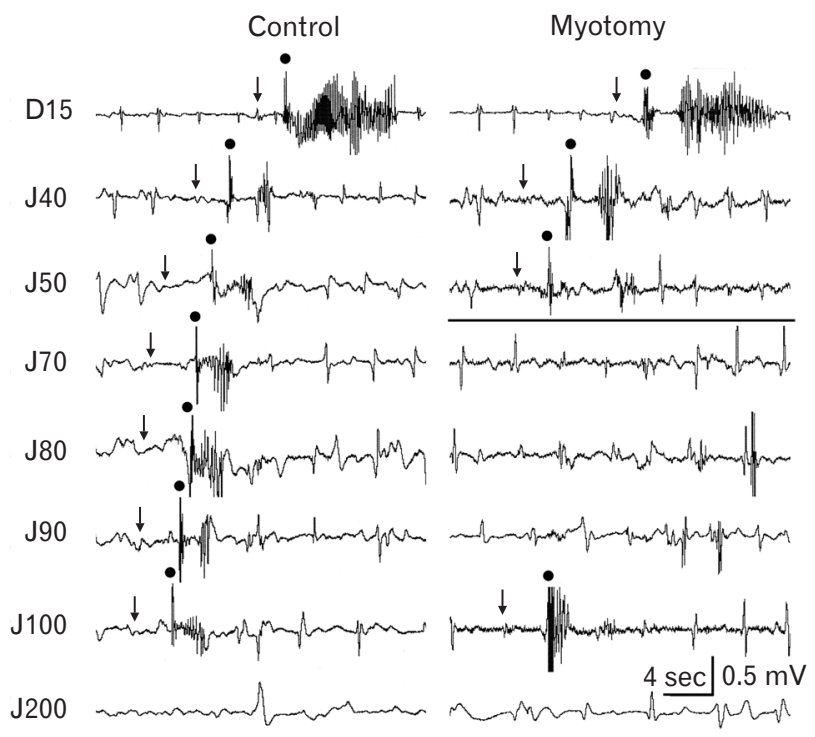

Figure 3. Effects of enteric nervous system (ENS) interruption on myoelectrical correlates of the retrograde giant contraction (RGC). This figure depicts the effects of myotomy at $60 \mathrm{~cm}$ from the pylorus on the initiation and propagation of electrical control activity (ECA) disruption and the RGC potential across the small intestine. Myotomy blocked the occurrence of the ECA disruption (the beginning of ECA disruption is indicated by the arrows) and RGC potential (indicated by dots above the RGC potential) at J70, J80, and J90, and reduced the delay between the RGC potential at J100 and J50 such that the RGC potential at J100 occurred at or after the RGC potential at J50. Myotomy had no observable effects orad to the myotomy. The horizontal line in this figure depict the location of the myotomy. D, duodenum; J, jejunum. The numbers after the letters indicate the $\mathrm{cm}$ from pylorus of the strain gauge. 
from the pylorus (J50), the length of intestine over which a denervation altered RGC or RGC potential, and the number of nonRGC contractions that occurred during the propagation of the RGC. Data was expressed as mean $\pm \mathrm{SE}$ and differences between means were tested using unpaired or paired Student's $t$ test. When the variances of the groups were not equal based on the $\mathrm{F}$ (variance ratio) test, non-parametric tests, ie, Wilcoxon signed-rank test, were used. Multiple comparisons of non-normally distributed data were tested using Kruskal-Wallis one-way analysis by ranks. A $P$-value of $<0.05$ was considered statistically significant for all tests.

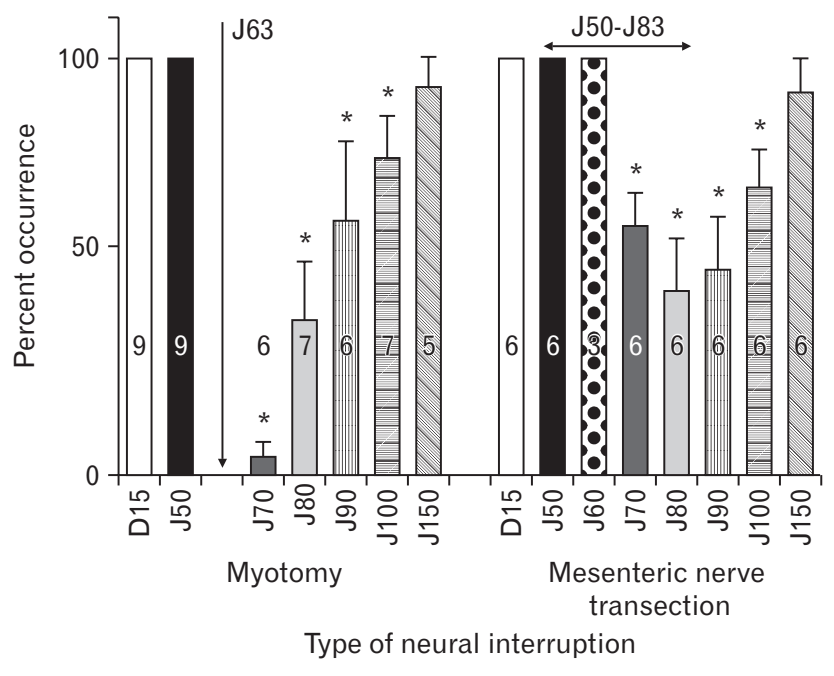

Figure 4. Effects of CNS or enteric nervous system (ENS) interruption on the percent occurrence of the retrograde giant contraction (RGC) or RGC potential (RGC-p). This figure depicts the effects of myotomy, ie, ENS interruption, or mesenteric nerve transection, ie, CNS interruption, on the percent occurrence of the RGC or RGC-p after apomorphine administration. Myotomy at $63 \pm 2 \mathrm{~cm}$ from the pylorus resulted in the significant reduction in the occurrence of the RGC or RGC-p from 20 to $50 \mathrm{~cm}$ aborad of the myotomy, and the effect was graded with the greatest effect closest to the myotomy. No effect orad of the myotomy was observed. Mesenteric nerve transection at $50 \pm 3$ to $83 \pm 6 \mathrm{~cm}$, ie, CNS interruption, caused no change in occurrence of the RGC or RGC-p up to $20 \mathrm{~cm}$ aborad within the denervated intestine, but reduced occurrence 20 to $50 \mathrm{~cm}$ aborad of the denervation. The maximum effect was at the most aborad location of the denervation and responses as far as $20 \mathrm{~cm}$ aborad of the most distal denervated intestine were also inhibited. No effect orad of the mesenteric nerve transection was observed. The numbers within the bars indicate the $\mathrm{n}$ for that group. ${ }^{*} \mathrm{P}<0.05$ for a difference from D15 using Kruskal-Wallace test. D, duodenum; J, jejunum. The numbers after the letters indicate the $\mathrm{cm}$ from pylorus of the strain gauge.

\section{Results}

\section{Effects of Myotomy}

Myotomy of the upper small intestine had significant effects on the RGC (Fig. 2) and its myoelectrical correlates (Fig. 3) activated by apomorphine, aborad but not orad, of the myotomy. The length of intestine over which the RGC or its myoelectrical correlates, ie, the RGC potential and ECA disruption, blocked due to myotomy was $17 \pm 5 \mathrm{~cm}(\mathrm{n}=9)$. The occurrence of the RGC or RGC potential was not only blocked aborad of the myotomy, but also the percent occurrences of the RGC and RGC potential at more aborad sites, up to $100 \mathrm{~cm}$ from the pylorus, were also reduced (Fig. 4).

The velocity of the RGC or RGC potential which propagated from J200 to D15 before myotomy was $12.1 \pm 0.8 \mathrm{~cm} / \mathrm{sec}(\mathrm{n}=$

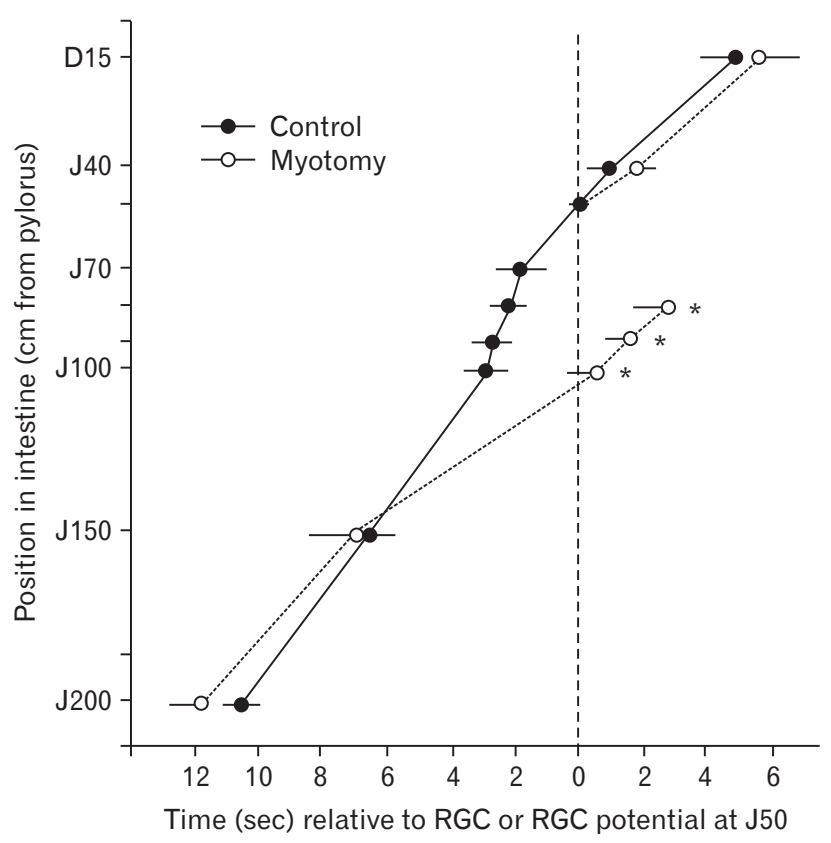

Figure 5. Effects of enteric nervous system (ENS) interruption on propagation of the retrograde giant contraction (RGC) or RGC potential across the small intestine. This figure depicts the effects of myotomy on the mean location of the RGC or RGC potential relative to the occurrence of the RGC or RGC potential at J50. Myotomy caused the RGC or RGC potential to slow its velocity from J150 to J80 such that the time delays from J100, J90, and J80 were significantly $\left({ }^{\star} P<\right.$ $0.05 ; \mathrm{n}=4$ to 8 ) different In addition, after myotomy 2 RGC's or RGC potentials occurred independent of one another on either side of the myotomy at J60. Myotomy had no significant effect on the RGC orad to the myotomy. D, duodenum; J, jejunum. The numbers after the letters indicate the $\mathrm{cm}$ from pylorus of the strain gauge. 
6), and after myotomy $11.6 \pm 0.8 \mathrm{~cm} / \mathrm{sec}(\mathrm{n}=5)$, which was not significantly different $(P>0.05)$. On the other hand, the propagation velocity of the RGC or RGC potential from J150 to J80 (J70 was blocked) decreased (Fig. 5), and from J100 to J80 (there were not enough values at $\mathrm{J} 150$ to statistically analyze the velocity from that location) decreased significantly $(P<0.05)$ from $18.1 \pm 3.0$ $\mathrm{cm} / \mathrm{sec}$ to $9.0 \pm 0.8 \mathrm{~cm} / \mathrm{sec}$. In addition, after myotomy the RGC or RGC potential occurring from J100 to J80 began after the RGC or RGC potential began at J50 (Fig. 2 and 5). That is, at the same time 2 independent RGC's or RGC potentials propagated simultaneously, 1 orad and 1 aborad of the myotomy.

The RGC's occurring aborad of the myotomy up to J100 were smaller (Fig. 2) in magnitude and duration. Because of the low numbers of animals $(n=5)$ for the myotomy group with strain gauges, the $\mathrm{N}$ for each intestinal location was insufficient for statistical purposes. In some cases the RGC was blocked further aborad

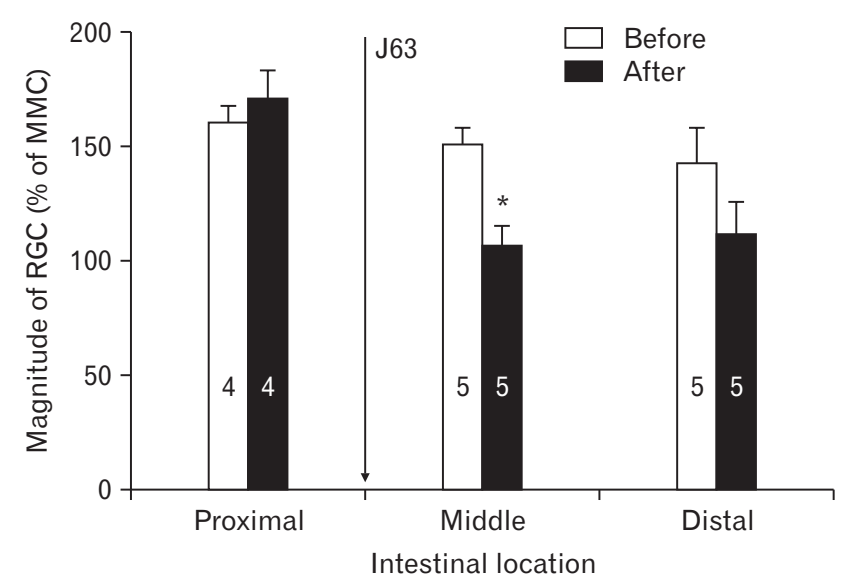

Figure 6. The effects of enteric nervous system (ENS) interruption on the magnitude of the retrograde giant contraction (RGC). This figure depicts the effects of myotomy at $63 \pm 2 \mathrm{~cm}$ from the pylorus, ie, ENS interruption, on the peak strain gauge response of the RGC relative to the peak strain gauge response of phase III of the migrating motor complex in the intestine orad, caudad within $50 \mathrm{~cm}$, and caudad distal to $100 \mathrm{~cm}$ of the myotomy. Myotomy significantly reduced the magnitude of the RGC within $50 \mathrm{~cm}$ aborad of the myotomy. ${ }^{*} \mathrm{P}<$ 0.05. MMC, migrating motor complex; J, jejunum. than J70, and in other cases the strain gauge was non-functional or not recorded. Therefore, the intestinal sites were combined into 3 sets of intestinal locations which were compared statistically: orad (locations or recording devices orad of the myotomy, ie, J50), aborad50 (locations of recording devices within $50 \mathrm{~cm}$ aborad of the myotomy, ie, J80, J90, and J100), and aborad100 (locations of recording devices at least $100 \mathrm{~cm}$ aborad of the myotomy, ie, J150 and J200). It was found in paired comparisons that while the magnitudes and durations of the RGC in the orad and aborad100 portion of the small intestine were not significantly affected by myotomy (Fig. 6 and 7), both magnitudes (Fig. 6) and durations (Fig. 7) of the RGC's in the aborad50 section of the small intestinal were significantly reduced.

Myotomy caused a significant myoelectrical change that may have contributed to the slowing of the RGC and RGC potential passing through this region, as described above. Myotomy signifi-

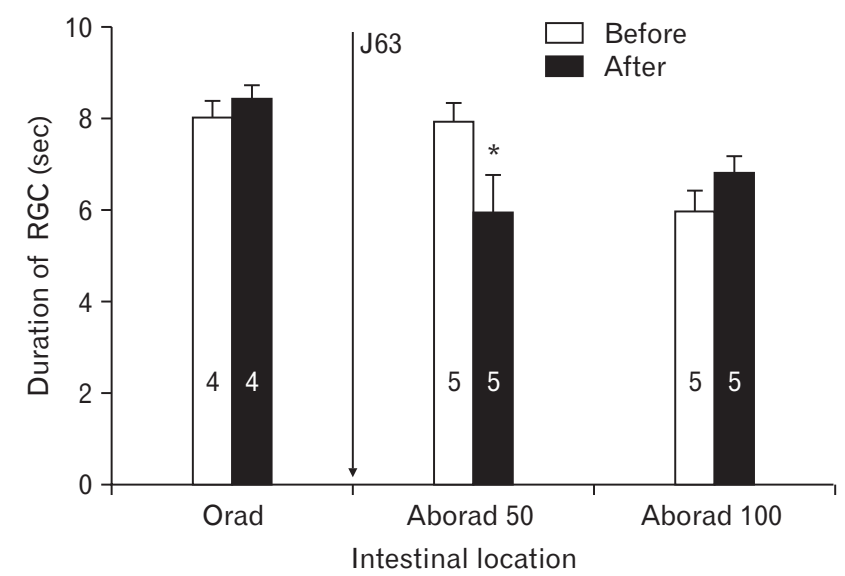

Figure 7. The effects of enteric nervous system (ENS) interruption on the duration of the retrograde giant contraction (RGC). This figure depicts the effects of myotomy at $63 \pm 2 \mathrm{~cm}$ from the pylorus, ie, ENS interruption, on the duration of the RGC the intestine orad, caudad within $50 \mathrm{~cm}$, and caudad distal to $100 \mathrm{~cm}$ of the myotomy. Myotomy significantly reduced the duration of the RGC within 50 $\mathrm{cm}$ aborad of the myotomy. ${ }^{*}<0.05$. J, jejunum.

Table. Effect of Myotomy at J60 on Electrical Control Activity Rate

\begin{tabular}{lcccccc}
\hline Intestinal location & J50 & J70 & J80 & J90 & J100 & J150 \\
\hline Control & $19.1 \pm 0.5$ & $19.0 \pm 0.7$ & $19.0 \pm 0.7$ & $18.7 \pm 0.5$ & $18.5 \pm 0.5$ & $14.6 \pm 0.6$ \\
Myotomy at J60 & $19.5 \pm 0.3$ & $14.3 \pm 0.5^{\mathrm{a}}$ & $14.3 \pm 0.4^{\mathrm{a}}$ & $14.7 \pm 0.5^{\mathrm{a}}$ & $14.7 \pm 0.5^{\mathrm{a}}$ & $14.2 \pm 0.5$ \\
\hline
\end{tabular}

${ }^{\mathrm{a}} \mathrm{P}<0.01$ for paired $t$ test $(\mathrm{n}=4)$.

$\mathrm{J}$, jejunum (the numbers after the letters indicate the $\mathrm{cm}$ from pylorus of the strain gauge).

Values are mean $\pm \mathrm{SE}$ of electrical control activity cycles per minute. 


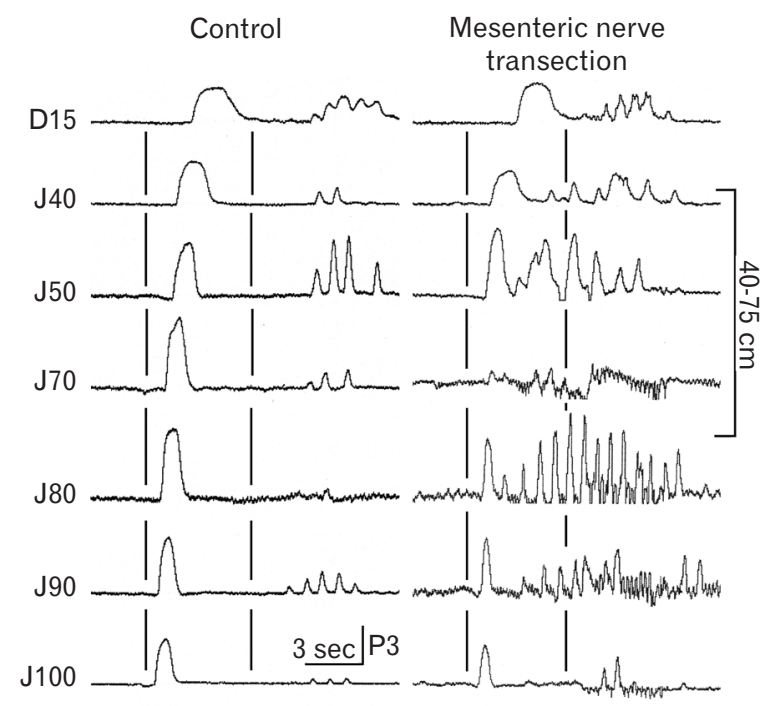

Figure 8. Effects of CNS interruption on retrograde giant contraction (RGC). This figure depicts the effects of mesenteric nerve transection at $\mathrm{J} 40$ to $\mathrm{J} 75 \mathrm{~cm}$ from the pylorus on the initiation, generation, and propagation of the RGC across the small intestine. Mesenteric nerve transection preserved the RGC over $10 \mathrm{~cm}$ from J40 to J50, blocked the $\mathrm{RGC}$ at $\mathrm{J} 70$, and reduced the magnitude and duration of the RGC at J80, J90, and J100. However, unlike myotomy the RGC sequence across the denervated intestine was not altered. That is unlike myotomy, an RGC potential aborad of the transection did not occur after initiation of the RGC orad of the denervation. Mesenteric nerve transection had no observable effects orad of the transected intestine. Mesenteric nerve transection also blocked the intestino-intestinal inhibitory reflex that allowed non-RGC contractions to occur during the propagation of the RGC, ie, between the vertical bars, which never occurred when the mesenteric nerve was intact. See Figure 2 for definition of symbols.

cantly reduced the ECA rate from 19 per minute to 14 per minute (Table) over the region of intestine just aborad of the myotomy, ie, J70 to J100 (Fig. 3).

\section{Effects of Mesenteric Nerve Transection}

Transection of the mesenteric nerves innervating a 30 to 40 $\mathrm{cm}$ segment of the proximal jejunum had significant effects on the RGC (Fig. 8) and its myoelectric correlates (Fig. 9) activated by apomorphine, aborad but not orad, of the denervation.

After mesenteric nerve transection, the occurrence of the RGC or RGC potential activated by apomorphine was unchanged in the first $16 \pm 4 \mathrm{~cm}$ of the denervated intestinal segment (up to $66 \pm$ $6 \mathrm{~cm}$ from the pylorus), but was reduced in magnitude (Fig. 8 and 10), duration (Fig. 8 and 11), or percent occurrence (Fig. 4, 8, and 9) up to $100 \pm 0 \mathrm{~cm}$ from the pylorus. The durations (Fig. 11) of

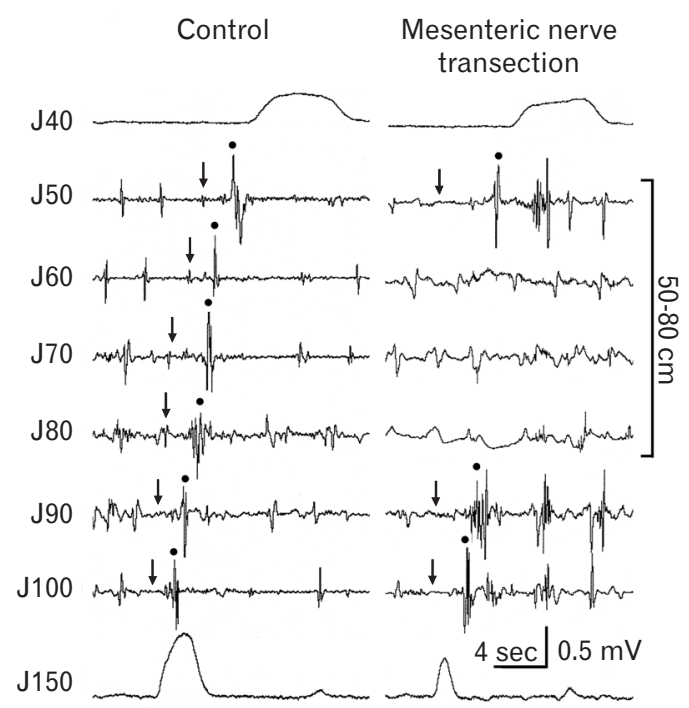

Figure 9. Effect of CNS interruption on myoelectrical correlates of the retrograde giant contraction (RGC). This figure depicts the effects of mesenteric nerve transection at 50 to $80 \mathrm{~cm}$ from the pylorus on the initiation, generation, and propagation of the RGC potential across the small intestine. Mesenteric nerve transection preserved the RGC potential and electrical control activity (ECA) disruption at J50, but blocked the occurrence of the RGC potential and ECA disruption at $\mathrm{J} 60, \mathrm{~J} 70$, and $\mathrm{J} 80$. In addition, mesenteric nerve transection reduced the delay of the RGC potential from J 90 and J100 to J50, but unlike myotomy, mesenteric nerve transection did not alter the sequence of activation of the RGC across the denervated intestine. That is unlike myotomy, an RGC potential aborad of the transection did not occur after initiation of the RGC orad of the denervation. See Figure 2 for definition of symbols.

the RGC were significantly shorter at J70 to J90 and of lower magnitude (Fig. 10) at J100. While these characteristics of the RGC between $65 \mathrm{~cm}$ and $100 \mathrm{~cm}$ from the pylorus were altered by interruption of CNS innervation of the intestine at $50 \pm 3$ to $83 \pm 6 \mathrm{~cm}$ from the pylorus, the propagation sequence of the $\mathrm{RGC}$ or RGC potential was not altered (Fig. 8 and 9). That is, there was only one $\mathrm{RGC}$ and it propagated orad in normal sequence.

Although the RGC and RGC potential propagated in normal sequence, they did not propagate at normal velocity after mesenteric nerve transection (Fig. 8 and 9). While the velocity of the RGC or RGC potential across the entire small intestine was not altered by interruption of CNS control $(13.3 \pm 1.6 \mathrm{~cm} / \mathrm{sec}[\mathrm{n}=5]$ before and $14.4 \pm 1.9 \mathrm{~cm} / \mathrm{sec}[\mathrm{n}=5]$ after, $P>0.05$ for paired $t$ test), the velocity of the RGC or RGC potential propagation over the denervated segment of the small intestine from J100 to J70 was significantly $(P<0.05, \mathrm{n}=6$, Wilcoxon signed-rank test) increased from $12.8 \pm 1.6 \mathrm{~cm} / \mathrm{sec}$ to $196 \pm 116 \mathrm{~cm} / \mathrm{sec}$ (Fig. 12). In 


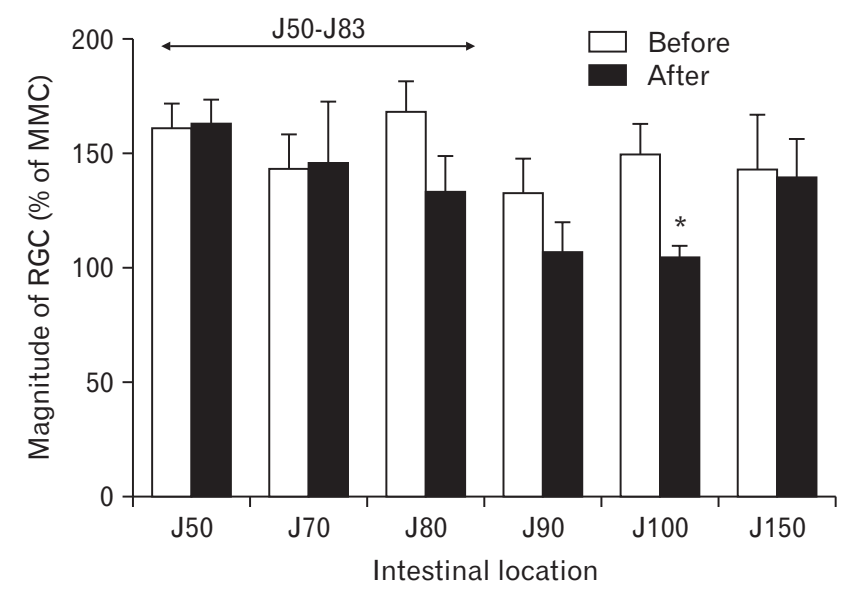

Figure 10. The effects of CNS interruption on the magnitude of the retrograde giant contraction (RGC). This figure depicts the effects of mesenteric nerve transection at $50 \pm 3$ to $83 \pm 6 \mathrm{~cm}$ from the pylorus, ie, CNS interruption, on the peak strain gauge response of the RGC relative to the peak strain gauge response of phase III of the migrating motor complex (MMC) in the intestine from J50 to J150. Mesenteric nerve transection significantly reduced the magnitude of the RGC at J100 which was almost $20 \mathrm{~cm}$ aborad of the denervated region. $\mathrm{n}=4$ for all groups. ${ }^{*} \mathrm{P}<0.05$. J, jejunum.

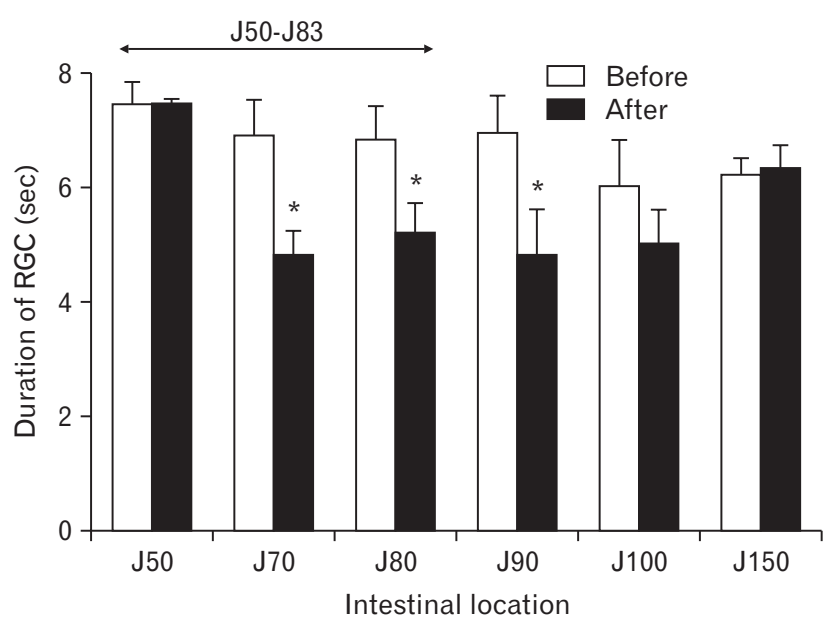

Figure 11. The effects of CNS interruption on the duration of the retrograde giant contraction (RGC). This figure depicts the effects of mesenteric nerve transection at $50 \pm 3$ to $83 \pm 6 \mathrm{~cm}$ from the pylorus, ie, CNS interruption, on the duration of the RGC of the intestine from J50 to J150. Mesenteric nerve transection significantly reduced the duration of the RGC from J70 to J90. $\mathrm{n}=4$ for all groups. ${ }^{*} \mathrm{P}<$ 0.05 . J, jejunum.

addition, the time delays from the occurrence of the RGC or RGC potential at J100, J90, J80, and J70 to J50 were significantly $(P<$ $0.05, \mathrm{n}=4$ to 8 ) reduced after interruption of the CNS control of the intestine by mesenteric nerve transection (Fig. 12).

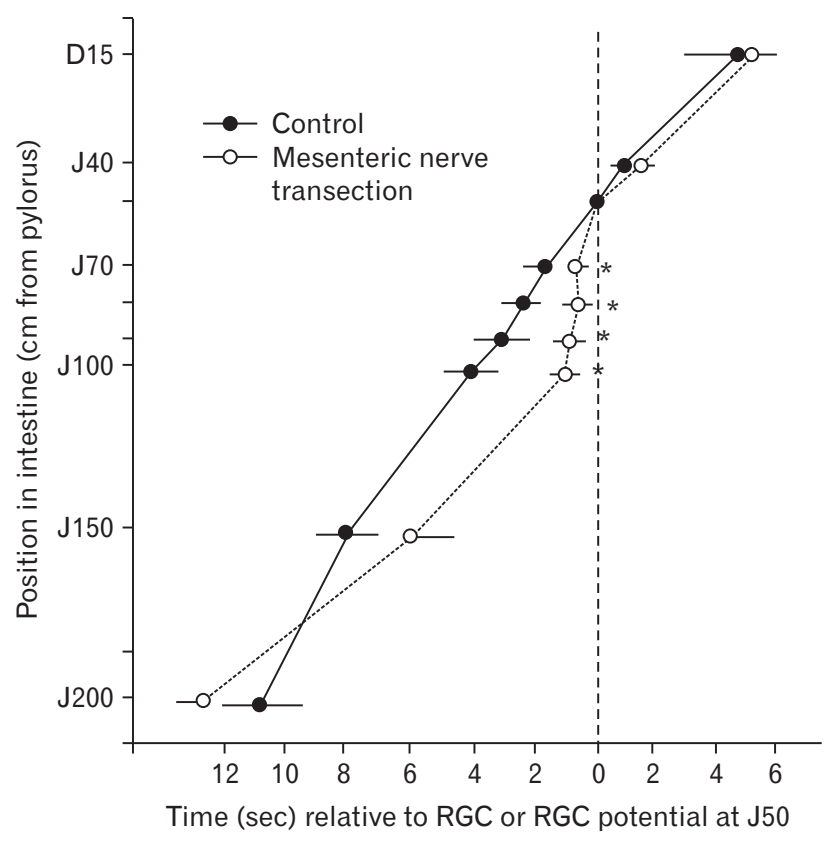

Figure 12. Effects of CNS interruption on the propagation of the retrograde giant contraction (RGC) or RGC potential across the small intestine. This figure depicts the effects of mesenteric nerve transection at $50 \pm 3$ to $83 \pm 6 \mathrm{~cm}$ from the pylorus on the mean location of the RGC or RGC potential relative to the occurrence of the RGC or RGC potential at J50. Mesenteric nerve transection caused the RGC or RGC potential to increase its velocity from J100 to J70 such that the time delays from these sites to $\mathrm{J} 50$ were significantly reduced $\left({ }^{\star} P<0.05 ; \mathrm{n}=4\right.$ to 8$)$. However, unlike myotomy, mesenteric nerve transection did not alter the sequence of activation of the RGC or RGC-p across the intestine. That is, 2 separate RGC's above and below the denervated section did not occur independent of each other. Mesenteric nerve transection had no significant effect on the RGC orad to the transection. J, jejunum.

Contractions of the small intestine other than the RGC did not occur during propagation of the RGC (Fig. 2 and 8), but after mesenteric nerve transection this absence of non-RGC contractions no longer occurred in the intestinal sites in which the neural supply had been interrupted (Fig. 8). The number of non-RGC contractions during the propagation of the RGC was $0 \pm 0$ at all intestinal locations under control conditions, and this significantly increased to $2 \pm 1$ at $\mathrm{J} 50(P<0.05, \mathrm{n}=4), 2 \pm 1$ at $\mathrm{J} 70(P<0.05, \mathrm{n}=4)$, $2 \pm 1$ at $\mathrm{J} 80(P<0.05, \mathrm{n}=4)$, and $2 \pm 0$ at $\mathrm{J} 90(P<0.05, \mathrm{n}=4)$, after mesenteric nerve interruption.

\section{Discussion}

The studies described in this manuscript have demonstrated that the mechanism of initiation, generation, and propagation of 


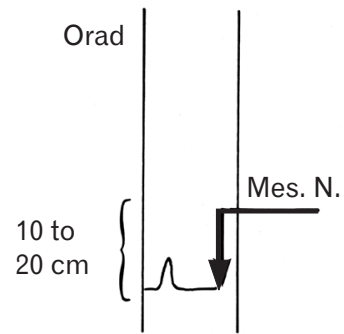

$\mathrm{i}_{1}$,

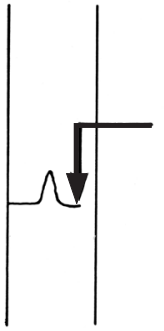

$\mathrm{i}_{2}$,

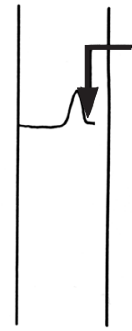

$\mathrm{i}_{\mathrm{n}}$
Figure 13. Mechanism of propagation of the retrograde giant contraction (RGC). This diagram shows the mechanism of propagation of the RGC. The CNS through the mesenteric nerve activates the RGC $10 \mathrm{~cm}$ to $20 \mathrm{~cm}$ aborad of the innervated site at its most aborad site, $i_{1}$, at mid small intestine. The CNS then activates the next orad site, $i_{2}$, in a similar manner and this continues until the RGC has completed its travel through the small intestine, $i_{n}$. Mes. N., mesenteric nerve.

the RGC is complex involving both the CNS and ENS. Interruption of either CNS or ENS innervation of segments of the small intestine did not block the orad progression or velocity of the RGC across the entire small intestine. This finding corroborates prior studies, ${ }^{5,10}$ and suggests that the extrinsic innervation, ie, the vagus nerves through the mesenteric nerves, ${ }^{5,6}$ control orad propagation of the RGC across the small intestine. However, other findings in this study demonstrate that this conclusion is incomplete. While neither myotomy nor mesenteric nerve transection altered orad propagation of the RGC across the entire small intestine, both surgeries had significant local effects on initiation, generation, and propagation of the RGC.

It was found that (1) after myotomy the RGC was absent for 10 to $20 \mathrm{~cm}$ aborad, but not orad, of the myotomy, and (2) after mesenteric nerve transection of a segment of the intestine the RGC was not absent from the first 10 to $20 \mathrm{~cm}$ of the extrinsically denervated region, but was inhibited aborad (but not orad) of this point. These findings suggest that the CNS through extrinsic innervation does not directly activate the innervated segment, but a segment of the intestine 10 to $20 \mathrm{~cm}$ aborad of the innervated site (Fig. 13). Whether the extrinsic neural pathway travels intramurally for 10 to $20 \mathrm{~cm}$ before synapsing with the ENS or whether the ENS propagates the neural input 10 to $20 \mathrm{~cm}$ aborad is unknown. However, while studies have identified long intramural nervous projections of extrinsic innervation in the esophagus, ${ }^{20}$ stomach, ${ }^{21}$ and distal colon, ${ }^{22}$ no such pathways were found in the proximal small intestine. ${ }^{23}$ On the other hand, studies indicate that most myenteric neurons project in an aborad direction. ${ }^{24,25}$ Therefore, it is possible that the aborad projection of ENS pathways may account for the aborad projection of the innervation that controls initiation of the RGC. This aborad projection of the ENS may also explain prior studies which found that the RGC was not observed in Thiry-Vella loops of intestine during emesis. ${ }^{11}$ Further studies are needed to confirm this hypothesis.

It was previously ${ }^{12}$ hypothesized that the extrinsic innervation initiates ECA disruption which leads to activation of the RGC by local factors. This conclusion was based on the finding that ECA disruption always preceded the RGC, and the velocity of the ECA was very consistent whereas the velocity of the RGC was highly variable. In addition, the maximum RGC velocity equaled ECA disruption propagation velocity. In the current study, the RGC potential was always preceded by ECA disruption regardless of the type of neural interruption. However, myotomy provided further evidence for the primacy of ECA disruption, rather than direct extrinsic control, in the activation of the RGC. Excluding the 10 to $20 \mathrm{~cm}$ of intestine aborad of the myotomy, which is under direct extrinsic neural control, myotomy should have no effect on initiation or propagation of the RGC if it is directly controlled by the CNS. However, after myotomy, 2 RGC's independent of each other occurred at the same time, one orad and the other aborad of the myotomy. These findings indicate that RGC initiation is not controlled directly by the CNS. Therefore, it is concluded that CNS controls initiation of ECA disruption and not the RGC. The generation of the RGC after initiation of ECA disruption is governed by local factors as discussed below.

The mechanism by which extrinsic innervation causes ECA disruption is unknown. The ECA is generated by the interstitial cells of Cajal (ICC) and the ICC is innervated by the ENS, ${ }^{5,16}$ therefore, it is likely that this ECA disruption is mediated by the ENS through the ICC. However, this study was not designed to examine this issue and cannot conclude anything in this regard.

One local factor altered by myotomy that may affect RGC initiation as well as propagation is the ECA rate. The ECA is a property of the intestinal smooth muscle and it represents the spontaneous fluctuation in the resting membrane potential of the muscle. ${ }^{19}$ The ECA of the small intestine is primarily generated by interstitial cells of Cajal (ICC) located in the muscularis. ${ }^{26}$ The ECA governs when a muscle contraction can occur. Contractions are possible when the membrane potential is in a depolarized state and is prevented when in a hyperpolarized state. Therefore, the rate of the ECA controls the rate of contraction. The ECA's of the intestinal muscles are not independent of each other. In the first quarter of the intestine, ie, the first 60 to $80 \mathrm{~cm}$ of the dog small intestine, ${ }^{19}$ the ECA of the muscle is tightly coupled such that the ECA's of all of the muscles fluctuate 
at the same rate but with a slight aborad delay. This tight electrical coupling is a property of the $\mathrm{ICC}^{26}$ which generates the ECA, and it guarantees that all contractions propagate aborally within a narrow range of velocities. Therefore, myotomy in the proximal jejunum, which transects the muscles of the seromuscular layer as well as the ICC that generates the ECA for these muscles, results in a significant decrease in the frequency and velocity of contractions aborad of the myotomy.

After myotomy, the RGC begins at the mid small intestine and propagates orad until it encounters the intestinal segment with a reduced $\mathrm{ECA}$ rate of $14 / \mathrm{min}$, at which point the RGC velocity falls from about $18 \mathrm{~cm} / \mathrm{sec}$ to $9 \mathrm{~cm} / \mathrm{sec}$. It is hypothesized that the slowed velocity of the RGC, through this region of the intestine, is due to the effects of myotomy on the ECA and its generator, the ICC. This slowed rate of propagation then results in the $\mathrm{RGC}$ arriving at its termination point just aborad (10 to $20 \mathrm{~cm}$ ) of the myotomy. By the time the RGC, aborad of the myotomy, reaches its termination point, the RGC orad of the myotomy has already been initiated by the CNS at normal velocity causing 2 RGC's on either side of the myotomy to occur simultaneously.

The mechanism responsible for the effect of mesenteric nerve transection on propagation of the RGC across the denervated segment is less clear. Mesenteric nerve transection, which had no effect on ECA rate, significantly increased the velocity of the RGC or RGC potential through the denervated region. This velocity was so fast that using strain gauges the RGC appeared to occur simultaneously. It is hypothesized that this rapid propagation of the RGC through this extrinsically denervated region of the intestine was due to local control mechanisms, ie, ENS and ICC, however, further studies are needed to define this effect.

The discussion above provides an understanding of the initiation and propagation of the RGC, but an understanding of the generation and magnitude of the RGC has not previously been presented. In a prior study ${ }^{13}$ comparing the effects of CCK and apomorphine in activating the RGC, it was found that $\mathrm{CCK}$ given intravenously activated the RGC at the same intestinal sites with the same magnitude and duration, as well as the same myoelectrical correlates as apomorphine. The only aspect that was not the same was that the RGC activated by CCK did not propagate. In addition, the effects of $\mathrm{CCK}$ were due to local neural elements as the response was not blocked by vagotomy or splanchnectomy, but was blocked by atropine. Therefore, prior studies indicated that the occurrence and magnitude of the RGC was dependent upon both the CNS and ENS. In the current study, the occurrence and magnitude of the RGC changed in the intestinal regions lacking either CNS or
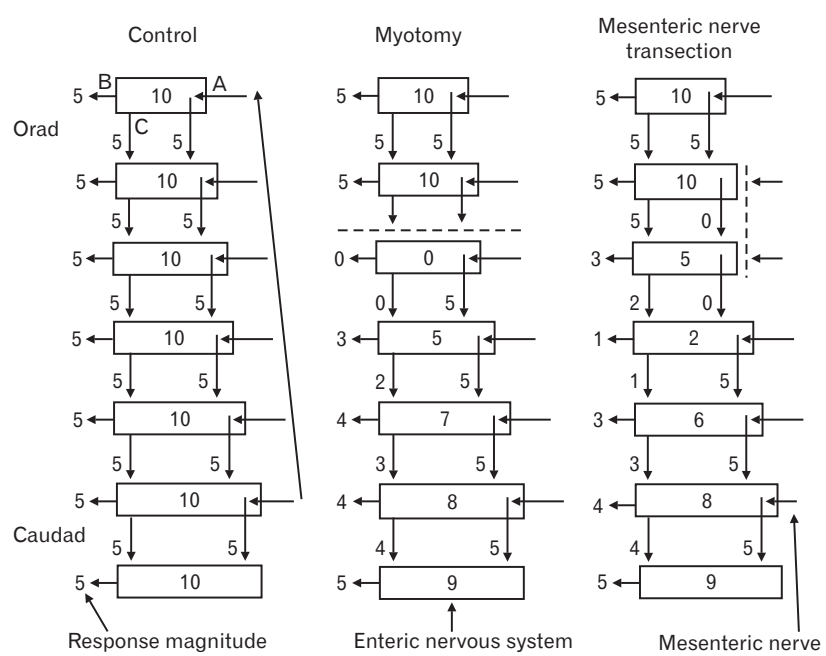

Figure 14. Model of the mechanism of retrograde giant contraction (RGC) generation. This figure depicts a model that assumes the following: (1) the CNS controls initiation of the RGC through the mesenteric nerves in a sequential fashion from caudad to orad (Fig. 13), (2) the mesenteric nerves provide innervation to enteric nervous system (ENS) to excite the RGC aborally, but not locally, and (3) the ENS communicates with itself primarily in an aborad direction. In this model, the ENS neurocircuitry that controls the RGC is organized into functional units portrayed as rectangular boxes. Each mesenteric input to the aborad ENS RGC functional unit supplies 5 units of excitement (A), and each ENS RGC functional unit provides equal units of whatever storehouse of excitement it has to the muscles to activate the RGC (B) and to activate the aborad ENS RGC functional unit (C). Under control conditions the most distal branch of the mesenteric nerve is activated first by the CNS and this excitement produces 5 units of excitement which activates the RGC just aborad of the innervation. The activation of the mesenteric nerve branch moves orad causing the more orad ENS RGC functional unit to produce 5 units of excitement to activate the RGC. This continues until the end of intestine. When a myotomy is performed the inter-ENS communications (inputs A and C) are lost resulting significant reduction in ENS output to the RGC at the next aborad ENS RGC functional unit causing total loss of the aborad RGC output (B). This loss in inter-ENS communication is projected aborally resulting in reduction in more aborad RGC outputs (B) but these recover in a graded fashion as extrinsic inputs (A) restore the lost excitation of ENS RGC functional units. Myotomy causes no deficits in an orad direction. When mesenteric nerve transection is performed, numerous ENS RGC functional units lose their CNS input (A) which causes the greatest RGC deficit to occur at the site just aborad to the most aborad ENS RGC functional unit extrinsically denervated. The most orad ENS RGC functional unit that lost its CNS input (A) as well as the functional units orad to the denervation exhibit no loss at all in RGC output (B). The RGC output (B) of aborad ENS RGC functional units are restored in a graded fashion as their excitation is replenished by CNS input (A). This model is a hypothesis which needs to be tested, but it does account for all of our research findings (Fig. 4, 6, 7, 10, and 11) regarding the occurrence and generation of the RGC. 
ENS control, indicating that the final motor response is a function of both the CNS and ENS. A hypothetical model accounting for all the effects on the generation and magnitude of the RGC observed in this study after interruption of either CNS or ENS, and based on basic physiology and anatomy of intestinal neural control, is presented in Figure 14.

The mesenteric nerves also mediate the intestine-intestinal inhibitory reflex (IIIR). The IIIR is activated by contraction or distension of the intestine and results in inhibition of the intestine at a distal site mediated by the mesenteric nerves. ${ }^{27}$ While this study was not designed to investigate the IIIR, the effects of cutting the mesenteric nerves on the IIIR were observed. It was found that transection of the mesenteric nerves significantly eliminated the absence of non-RGC contractions during the propagation of the RGC at the sites where the mesenteric nerve was transected, ie, 50 to $80 \mathrm{~cm}$ from the pylorus, and for $10 \mathrm{~cm}$ aborad of the transection. It is likely that, under control conditions, the absence of non-RGC contractions during the propagation of the $\mathrm{RGC}$ was due to activation of the IIIR by the propagating RGC, as cutting the mesenteric nerves which mediate this reflex removed this inhibition.

In summary, these studies found that the initiation, generation, and propagation of the RGC associated with vomiting are controlled by both the CNS through the mesenteric nerves, and the ENS. These studies indicate that the CNS initiates the orad sequence of ECA disruption, which eliminates the primary obstacle for orad transport, ie, ECA. Disruption of the ECA establishes a window of opportunity for the occurrence of the RGC, but the generation of the RGC within this window and its magnitude depend primarily on the ENS. The mechanism of ECA disruption is unknown, but it is likely a result of extrinsic neural control of the ICC.

Acknowlegements: The support of Dr Sushil Sarna in the formulation of these studies and the technical assistance of Jeffrey Marvig were greatly appreciated.

Financial support: This study was supported by Veterans Affairs Merit Review Grant 5120-02P.

\section{Conflicts of interest: None.}

\section{References}

1. Lang IM, Marvig J. Functional localization of specific receptors mediating the GI motor correlates of vomiting. Am J Physiol 1989;256(Pt 1): G92-G99.
2. Alvarez, WC. Reverse peristalsis in the bowel, a precursor of vomiting. JAMA 1925;85:1051-1054.

3. Ehrlein, HJ. Retroperistaltism and duodenogastric reflux in dogs. Scand J Gastroenterol Suppl 1981;67:29-32.

4. Aizawa, I, Negishi K, Suzuki T, Itoh Z. Gastrointestinal contractile activity associated with vomiting in the dog. In: C Roman, ed. Gastrointestinal Motility. Lancaster: MTP Press 1984:159-164.

5. Lang IM, Sarna SK, Condon RE. Gastrointestinal motor correlates of vomiting in the dog: Quantification and identification as an independent phenomenon. Gastroenterology 1986;90:40-47.

6. Lang IM, Schnepf KJ. Identification of the abdominal neural pathway mediating the intestinal motor correlates of vomiting. In: Bianchi AL, Grelot L, Miller AD, King GL, eds. Mechanism and control of emesis. Montrouge: John Libbey Eurotext 1992:81-82.

7. Berthoud HR, Carlson NR, Powley TL. Topography of efferent vagal innervation of the rat gastrointestinal tract. Am J Physiol 1991;260(Pt 2):R200-R207.

8. Berthoud HR, Powley TL. Characterization of vagal innervation to the rat celiac, suprarenal and mesenteric ganglia. J Auton Nerv Syst 1993;42: 153-169.

9. Woods JD. Enteric nervous system: the brain-in-the-gut. In: Granger DN, Granger JP, eds. Colloquium series on integrative systems physiology from molecule to function to disease. San Rafael: Morgan \& Claypool Life Sciences 2011.

10. Sha S, Matsushima Y, Habu S, Mishima Y, E Okamoto. Extrinsic nervous control of retrograde giant contraction during vomiting in conscious dog. Dig Dis Sci 1996;41:1546-1550.

11. Gregory RA. Changes in intestinal tone and motility associated with nausea and vomiting. J Physiol Lond 1946;105:58-65.

12. Lang IM, Marvig J, Sarna SK, Condon RE. The gastrointestinal myoelectrical correlates of vomiting in the dog. Am J Physiol 1986;251(Pt 1): G830-G838.

13. Lang IM, Marvig J, Sarna SK. Comparison of the gastrointestinal responses to CCK-8 and associated with vomiting. Am J Physiol 1988;254(Pt 1):G254-G263.

14. Borison HL, Wang SC. Functional localization of the central coordinating mechanisms for emesis in cat. J Neurophysiol 1949;12:305-313.

15. Borison HL. Area postrema: chemoreceptor circumventricular organ of the medulla oblongata. Prog Neurobiol 1989;32:351-390.

16. Lang IM, Sarna SK. Motor and myoelectric activity associated with vomiting, regurgitation and nausea. In: Wood JD, ed. Handbook of Physiology, Gastrointestinal system, motility and circulation. Bethesda, MD: American Physiological Society 1989:1179-1198.

17. Lang, IM, Sarna SK, Shaker R. Gastrointestinal motor and myoelectrical correlates of motion sickness. Am J Physiol 1999;277(Pt 1):G642-G652.

18. Lang, IM, Sarna SK, Dodds WJ. The pharyngeal, esophageal, and gastric responses associated with vomiting. Am J Physiol 1993;265(Pt 1):G963-G972.

19. Sarna SK. In vivo myoelectric activity, methods, analysis, and interpretation. In: Wood JD, ed. Handbook of physiology, gastrointestinal motility and circulation. Bethesda, MD: American Physiological Society 1989:817-864. 
20. MacGilchrist AJ, Christensen J, Rick GA. The distribution of myelinated nerve fibers in the mature opossum esophagus. J Auton Nerv Syst 1991;35:227-235.

21. Christensen J, Rick GA. Shunt fascicles in the gastric myenteric plexus in five monogastric species. Gastroenterology 1985;88:1020-1025.

22. Christensen J, Stiles MJ, Rick GA, Sutherland J. Comparative anatomy of the myenteric plexus of the distal colon in eight mammals. Gastroenterology 1984;86:706-713.

23. Christensen J. Regional variations in the gross structure of the enteric nervous systems: some new views. Trans Am Clin Climatol Assoc 1990;101:21-36; discussion 36-37.
24. Brookes SJ. Classes of enteric nerve cells in the guinea pig small intestine. Anat Rec 2001;262:58-70.

25. Furness JB. Types of neurons in the enteric nervous system. J Auton Nerv Syst 2000;81:87-96.

26. Blair, PJ, Rhee PL, Sanders KM, Ward SM. The significance of interstitial cells in neurogastroenterology. J Neurogastroenterol Motil 2014;20:294-317.

27. Frantzides CT, Sarna SK, Matsumoto T, Lang IM, Condon RE. An intrinsic neural pathway for long intestino-intestinal inhibitory reflexes. Gastroenterology 1987;92:594-603. 